This report was propared as an account of work sponsored by an agency of the United States Government. Neither the United States Government nor any agency thereof, nor any of their employees, makes any warranty, express or implied, or assumes any legal liability or responsibility for the accuracy, completeness, or usefulness of any information, apparatus, product, or process disclosed, or represents that its use would not infringe privately owned rights. Reference herein to any specific commercial product, process, or service by trade name, trademark, manufacturer, or otherwise does not necessarily constitute or imply its endorsement, recommendation, or favoring by the United States Government or any agency thereof. The views and opinions of authors expressed herein do not necessarily state or reflect those of the United States Government or any agency thereof.

\title{
EXPERIMENTAL RESULTS FROM CERN ON REACTION MECHANISMS IN HIGH ENERGY HEAVY ION COLLISIONS
}

Soren P. SORENSEN

Dept. of Physics, University of Tennessee, Knoxville, TN 37996, USA and

Oak Ridge National laboratory, P. O. Box 2008, Oak Ridge, TN 37831-6374, USA

Three main experimental results from CERN concerning seaction mechanisms in high Three main experimental results from CERN concerning reaction the single particle picture, 2) the nuclear stopping power and 3) the attained energy densities.

\section{INTRODUCTION}

One of the primary goals of the initial phase of the CERN heavy ion program has been to study the reaction $n$-chanisms of ultrarelativistic heavy ion collisions in order to establish a baseline against which any QGP signal will have to be evaluated. Three collaborations, NA34, NA35, and WA80, have to a significant extend incorporated detectors sensitive to global parameters in their setups. The most important recorded quantities have been: 1) charged particle multiplicity, 2) transverse energy and 3) forward energy. For 1) and 2) also pseudorapidity distributions have been measured. Due to the limited space available it is not possible here to present the raw experimental data. Please refer to refs. 1 and 2 for a more detailed review. Instead we will here focus on three of the more important results derived from the data.

\section{VALIDITY OF THE SINGLE PARTICLE PICTURE}

The most important parameter for understanding the features of minimum bias data has turned out to be the nuclear overlap volume or, with other words, the number of participating

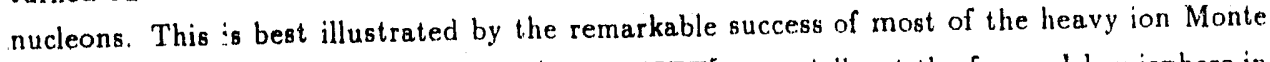
Carlo models, like FRITIOF ${ }^{3}, V_{E N U S}^{4}$ and HIJET ${ }^{5}$, especially at the forward hemisphere in the CMS, where the codes in general are within $10-15 \%$ of the data. These codes are all to first order based on a linear superposition of individual collisions between the participating nucleons. This simple pictare bets, however, more complicated at smaller pseudorapidities, where rescattering effects ir. the larget spectator matter can be expected to contribute. Only models like MCFM ${ }^{6}$, HIJET, VENUS, and RQMD ${ }^{7}$, which in various ways incorporate rescattoring, are able tc obtain resonable fit to tratsverse energy data recorded in the target rapidity repion. At the moment the detailed investigation of these rescattering effects seems to be the most promising tool for extracting information on the particle formation time $\tau_{0}$. Current estimates ${ }^{4-7}$ indicate a value of $\tau_{o}$ around $1.2 \mathrm{fm} / \mathrm{c}$. The systematic uncertainties are, however, large. Especially the transverse mass dependence of $\tau_{n}$ seems to be uncertain.

Currently no generally accepted collective phenomena have been discovered. Flow analysis, that has been very successful at Bevalac energies, has given negative results. The shapes 
of pion transverse momentum distributions have been interpreted as evidence of collective transverse flow ${ }^{8}$, but that conclusion has not been generally accepted ${ }^{\theta}$.

\section{NUCLEAR STOPPING POWER}

Estimates of the nuclear stopping power in heavy ion collisions have been difficult to obtain due to a proliferation of definitions of the concept. Based on leacing baryon spectra the momentum degradation length in $\mathrm{p}+\mathrm{A}$ reactions has been determined to be $6 \pm 2 \mathrm{fm}^{10}$ corresponding to a rapidity shift of 2.4 in central $\mathrm{p}+\mathrm{Pb}$ collisions. Based on the available $\mathrm{E}_{\mathrm{T}}$ and forward energy - spectra Wong determined ${ }^{11}$ the effective momentum degradation length in ${ }^{18} \mathrm{O}$-induced reactions at 60 and $200 \mathrm{~A} \mathrm{GeV}$ to be approximately $4 \mathrm{fm}$ indicating a very large degree of stopping in nuclear collisions. Another approach ${ }^{12}$ based on the Landau fireball model has estimated the total energy contained in the fireball to be around $90 \%$ of the available CM energy consistent with a momentum degradation length of $5 \mathrm{fm}$. It should be noted that an energy independent momentum degradation length, as would be expected by the constant nucleon-nucleon cross section at $\sqrt{s}>3 \mathrm{GeV}$, will result in a constant ratio between the fireball energy and the CM energy. This does, however, not necessarily indicale full stopping, which should be defined as the situation where the average final rapidities of the projectile and target participants are the same. The "mid-rapidity stopping", defined as $\left(d \mathrm{E}_{\mathrm{T}} / d \eta\right)_{\max } /\left(E_{\mathrm{cm}} / 2\right)$, has been introduced by $\mathrm{WA} 80^{13}$ as a simple and convenient experimental measure of stopping. Table 1 illustrates how this quantity increases with decreasing beamenergy and increasing target mass in central collisions.

\section{ENERGY DENSITY}

Most simple estimates of the attained energy densities have been based on two different approaches. In the first the global energy density has been defined as $\epsilon=$ (some energy) / (some volume). Typically the energy has been chosen as the total transverse energy and the volume as the Lorentz-contracted target volume, but many other combinations have been used. The fundamental problem with this approach, which in general leads to $\epsilon=3.8 \mathrm{GeV} / \mathrm{fm}^{3}$, is that at the time during the reaction where the fireball volume is as small as the $f$. ex. the target nucleus, then only a fraction of the total fireball energy has come on the mass shell due to timedilated formation times. In short, the energy and volume are not refering to the same time. A more realistic estimate of the on-shell energy at the point of

\begin{tabular}{|l||c|c|c|c|c|}
\hline$\Downarrow$ Reaction $\mid$ Target $\Rightarrow$ & $\mathrm{C}$ & $\mathrm{Al}$ & $\mathrm{Cu}$ & $\mathrm{Ag}$ & $\mathrm{AU}$ \\
\hline $60 \mathrm{~A} \mathrm{GeV}^{16} \mathrm{O}$ & 0.29 & & 0.37 & 0.39 & 0.44 \\
$200 \mathrm{~A} \mathrm{GeV}^{16} \mathrm{O}$ & 0.22 & & 0.27 & 0.31 & 0.34 \\
$200 \mathrm{~A} \mathrm{GeV}^{32} \mathrm{~S}$ & & 0.22 & 0.24 & 0.25 & 0.29 \\
\hline
\end{tabular}

Table 1: Mid-rapidity stopping measure for extracted from WA80 data. Please see text for further information 
time during the collision, where the fireball volume has a size close to the target participant volume, will therefore lead to considerable lower energy densities.

The other approach has been to use Bjorkens ${ }^{14}$ fnrmula, which leads to values of a around $3 \mathrm{GeV} / \mathrm{fm}^{3}$ for the most central $\mathrm{O}+\mathrm{Au}$ collisions assuming $\tau_{0}=1 \mathrm{fm} / \mathrm{c}$. The basic problem in applying Bjorkens formula at $200 \mathrm{~A} \mathrm{GeV}$ is not so much the lack of cylindrical boostinvariance, but more the ignorance of the nuclear transit time, which is close $t 01 \mathrm{fm} / \mathrm{c}$. The kinetic energy of the nuclei is therefore not instantaneously transformed into off-shell gluon field energy, but the energy release takes place gradually during the transit time. This will again le ad to lower values of the maximum energy density.

Probably the most detailed study of different formation- and reaction-times and their influence on the attained energy density can be found in ref. 15. Here it is found, based on a space-time version of ATTILA, that the maximum energy density in central $200 \mathrm{~A}$ (ieV $\mathrm{O}+\mathrm{Au}$ collisions is only around $1.5 \mathrm{GeV} / \mathrm{fm}^{3}$.

\section{CONCLUSIONS}

At CERN SPS energies heavy ion Monte Carlo codes has reached a degree of maturity, where global parameters like transverse energy and charged particle multiplicitics can be predicted to within $10-15 \%$. The nuclear stopping power seems to be high as expressed in the low value of 4-5 $\mathrm{fm}$ for the momentum degradation length, but the attained maximum energy density in central collisions is probably not higher than $1.5 \mathrm{GeV} / \mathrm{fm}^{3}$, which might be just short of the energy density needed for the phase transition beween the hadron and the quarkgluon pliases.

\section{REFERENCES}

1) J. Stachel, Review of experimental results from the AGS program, this volume

2) H. Specht, Review of experimental results from the CERN program, this volume

3) B. Anderson et al., Nucl. Phys. B281 (1987) 289

4) K. Werner, Z. Phys. C42 (1989) 85

5) A. Shor and. R. Longacre, Phys. Lett. B218 (1989) 100

6) J. Ranft, Z. Phys. C43 (1989) 439

7) H. Sorge, H. Stocker and W. Greiner, Nucl. Phys. A498 (1989) 567c

8) K. S. Lee and U. Heinz, Z. Phys. C43 ( 1989) 425

9) D. Kusnezov and G. Bertsch, Phys. Rev. C40 (1989) 2075

10) W. Busza and R. Ledoux, Ann. Rev. Nucl. Part. Phys. 38 (1988) 119

11) C.-Y. Wong, Phys. Rev D39 (1989) 2606

12) J. Stachel and P. Braun-Munzinger, Phys. Lett. B2.16 (1989) 1

13) WA80 Collaboration, R. Albrecht et al., Phys. Lett. B199 (1987) 45

14) J. D. Bjorken, Phys. Rev, D27 (1982) 140

15) M. Gyulassy and M. Plumer, LBL-27885 preprint (1989) 

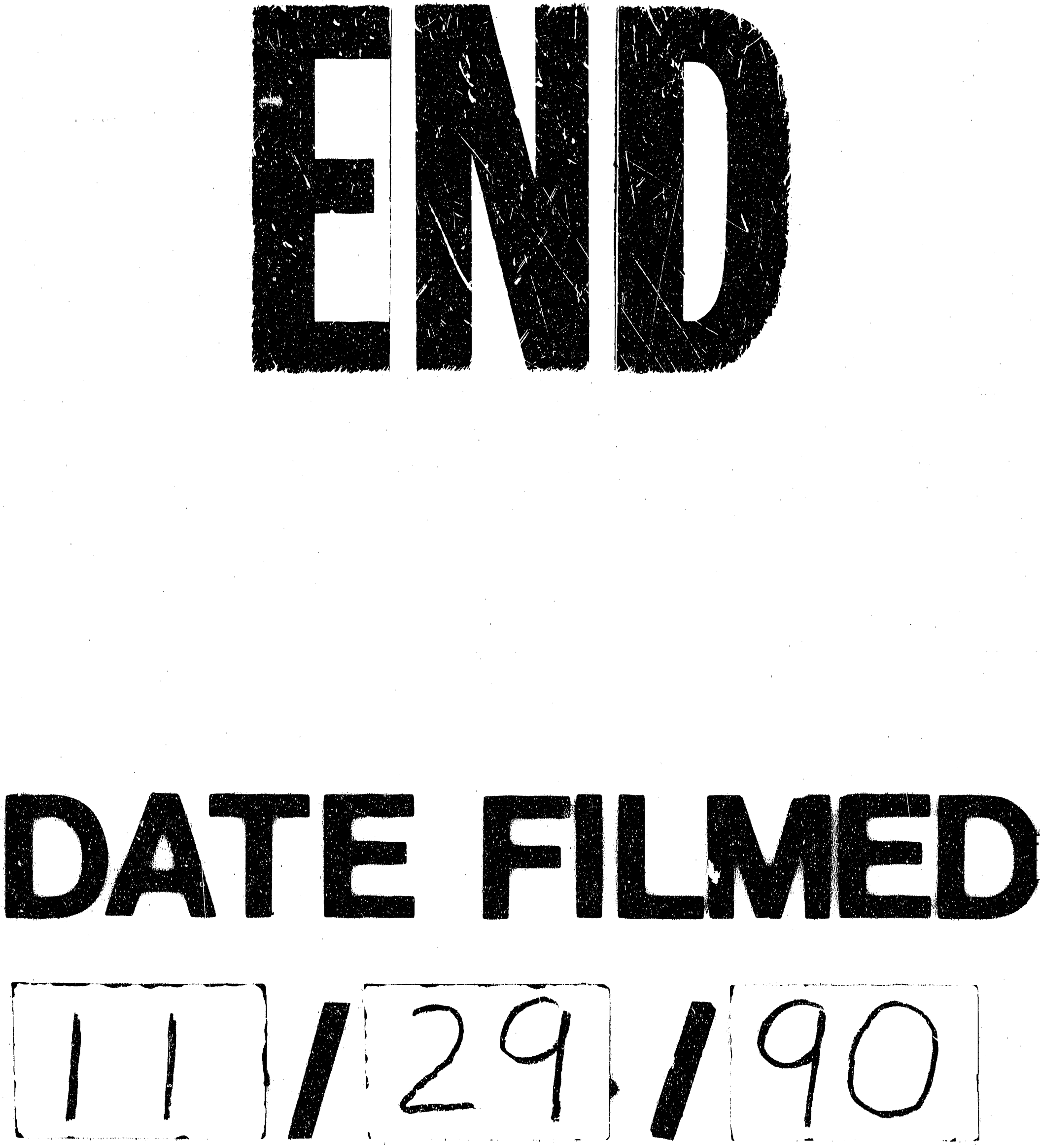
